\title{
AN ANALOGUE OF HARDY'S THEOREM FOR VERY RAPIDLY DECREASING FUNCTIONS ON SEMI-SIMPLE LIE GROUPS
}

\author{
A. Sitaram AND M. Sundari
}

We generalise a result of Hardy, which asserts the impossibility of a function and its Fourier transform to be simultaneously "very rapidly decreasing", to: (i) all noncompact, semi-simple Lie groups with one conjugacy class of Cartan subgroups; (ii) $S L(2, \mathbb{R})$; and (iii) all symmetric spaces of the noncompact type.

\section{Introduction.}

A celebrated theorem of $\mathrm{L}$. Schwartz asserts that a function $f$ on $\mathbb{R}$ is 'rapidly decreasing' (or in the 'Schwartz class') iff its Fourier transform is 'rapidly decreasing'. Since this theorem is of fundamental importance in harmonic analysis, there is a whole body of literature devoted to generalizing this result to other Lie groups. (For example, see [18].) In sharp contrast to Schwartz's theorem, is a result due to Hardy [5] which says that $f$ and $\hat{f}$ cannot both be "very rapidly decreasing". More precisely, if $|f(x)| \leq A e^{-\alpha|x|^{2}}$ and $|\hat{f}(y)| \leq$ $B e^{-\beta|y|^{2}}$ and $\alpha \beta>\frac{1}{4}$, then $f \equiv 0$. (See [2], pp. 155-157.) However, as far as we are aware, until very recently no systematic attempt was made to generalize Hardy's theorem to other Lie groups. In [12], [13], and [15], this result has been generalized to the Heisenberg groups $\mathbb{H}_{n}$, the Euclidean motion groups $M(n)$ and for certain eigenfunction expansions. In this paper we establish an analogue of Hardy's theorem for a class of noncompact semisimple Lie groups and all symmetric spaces of the noncompact type.

Hardy's theorem can also be viewed as a sort of 'Uncertainty Principle'. The results in [12] and [13] are presented from this point of view.

(In [1], Cowling and Price have proved an " $L^{p}-L^{q}$ " version of Hardy's theorem on $\mathbb{R}$. The theorem of Beurling in [9] is similar in spirit to Hardy's theorem, although far more general, and indeed Hardy's theorem, as well as the result of Cowling and Price, can be deduced from it as special cases.)

\section{Notation and Preliminaries.}

Let $G$ be a connected, non-compact, semi-simple Lie group with finite centre and $K$ a fixed maximal compact subgroup of $G$. Let $\mathcal{G}, \mathcal{K}$ denote the Lie 
algebras of $G$ and $K$ respectively. Suppose $\mathcal{G}=\mathcal{K} \oplus \mathcal{P}$ is a Cartan decomposition of $\mathcal{G}$ and $B$ is the Cartan-Killing form of $\mathcal{G}$. It is known that $B$ restricted to $\mathcal{P}$ is positive definite. Therefore $B$ defines an inner product on the real vector space $\mathcal{P}$. Let $P=\exp \mathcal{P}$. Then $G$ is diffeomorphic to $K \times P$ under the map $(k, u) \mapsto k u$ for $k \in K$ and $u \in P$. Therefore each $g \in G$ can be uniquely written as $g=g_{K} g_{P}$ with $g_{K} \in K$ and $g_{P} \in P$. Since $P$ and $\mathcal{P}$ are diffeomorphic under the exponential map, $g_{P}=\exp X$ for a unique $X \in \mathcal{P}$. Define $\|g\|_{G}=B(X, X)^{\frac{1}{2}}$.

Fix a maximal abelian subspace $\mathcal{A}$ of $\mathcal{P}$. Let the dimension of $\mathcal{A}$ be $l$.(' $l$ ' is called the real rank of $\mathcal{G}$.) The restriction $\left.B\right|_{\mathcal{A} \times \mathcal{A}}$ gives an inner product on $\mathcal{A}$ and we can identify $\mathcal{A}$ with $\mathbb{R}^{l}$ under this inner product. Let $\Delta$ denote the set of roots for the adjoint action of $\mathcal{A}$ on $\mathcal{G}$. Fix a Weyl-chamber $\mathcal{A}^{+}$of $\mathcal{A}$ and let $\Delta^{+}$be the corresponding set of positive roots (see [7] for details). Let $A=\exp \mathcal{A}$ and $A^{+}=\exp \mathcal{A}^{+}$. If $\overline{A^{+}}$denotes the closure of $A^{+}$in $G$ then it is known that $G=K \overline{A^{+}} K$, the polar decomposition of $G$ i.e. each $x \in G$ can be written as $x=k_{1} a k_{2}$, for $k_{1}, k_{2} \in K$ and $a \in \overline{A^{+}}$. If $\mathcal{G}_{\alpha}$ denotes the root space corresponding to $\alpha \in \Delta$, then we can choose a Haar measure $d x$ on $G$ such that relative to the polar decomposition it is given by $d x=J(a) d k_{1} d a d k_{2}$ where $J(a)=\prod_{\alpha \in \Delta^{+}}\left(e^{\alpha(\log a)}-e^{-\alpha(\log a)}\right)^{n(\alpha)}$, $n(\alpha)=\operatorname{dim} \mathcal{G}_{\alpha}$ and ' $l o g$ ' is the inverse of the map 'exp' on $\mathcal{A}$ i.e. $\int_{G} f(x) d x=$ $\int_{K} \int_{\overline{A^{+}}} \int_{K} f\left(k_{1} a k_{2}\right) J(a) d k_{1} d a d k_{2}$, where $d a$ is the Haar measure on $A$. Let $G=K A N$ be the corresponding Iwasawa decomposition of $G$ (see [7] for details). The Iwasawa decomposition gives rise to the projection mappings $\kappa: G \rightarrow K, a: G \rightarrow A$, and $n: G \rightarrow N$. Then we have

$$
x=\kappa(x) \exp H(x) n(x),
$$

where $\kappa(x) \in K, H(x) \in \mathcal{A}, H(x)=\log a(x), n(x) \in N$.

If $M$ denotes the centralizer of $A$ in $K$ then $P=M A N$ is the minimal parabolic subgroup of $G$. Fix $\xi \in \widehat{M}$ and let $\mathbb{H}_{\xi}$ be the finite dimensional Hilbert space on which $\xi$ acts, $d(\xi)=\operatorname{dim} \mathbb{H}_{\xi}$. For $\lambda \in \mathcal{A}^{*}$ (the real dual of $\mathcal{A})$, define a representation $(\xi, \lambda)$ of $P$ by:

$$
(\xi, \lambda)(\operatorname{man})=\xi(m) \exp ((\imath \lambda+\rho)(\log a)),
$$

where $\log : A \rightarrow \mathcal{A}$ is the inverse of the map exp : $\mathcal{A} \rightarrow A$ and $\rho=$ $\frac{1}{2} \sum_{\alpha \in \Delta^{+}} n(\alpha) \alpha, m \in M, a \in A, n \in N$. From this representation we get, by induction, a representation $\pi_{\xi, \lambda}$ of $G$ acting on the Hilbert space

$$
\begin{array}{r}
\mathbb{H}_{\xi}^{o}=\left\{g: K \rightarrow \mathbb{H}_{\xi} \text { measurable }: g(k m)=\xi\left(m^{-1}\right) g(k), \quad k \in K, m \in M\right. \\
\text { and } \left.\int_{K}\|g(k)\|^{2} d k<\infty\right\}
\end{array}
$$


where $\|\cdot\|$ denotes the norm on $\mathbb{H}_{\xi}$. The induced representation $\pi_{\xi, \lambda}$ acts unitarily on $\mathbb{H}_{\xi}^{o}$ by the formula

$$
\left(\pi_{\xi, \lambda}(x) g\right)(k)=e^{-(\imath \lambda+\rho)\left(H\left(x^{-1} k\right)\right)} g\left(\kappa\left(x^{-1} k\right)\right)
$$

for $x \in G, k \in K, g \in \mathbb{H}_{\xi}^{o}$. Note that the action of $K$ on $\mathbb{H}_{\xi}^{o}$ is just the left regular action.

Given $\xi \in \widehat{M}$, it is known that one can find a dense open subset $O_{\xi}$ of $\mathcal{A}^{*}$ such that $\pi_{\xi, \lambda}$ is irreducible for all $\lambda \in O_{\xi}$ (see [10], pp. 174 for details). Let $W$ be the Weyl group of the pair $(\mathcal{G}, \mathcal{A})$. Then there is a natural action of $W$ on $\widehat{M} \times \mathcal{A}^{*}$ and the only identifications among the irreducible representations in these series of representations are the identifications given by the Weyl group action (see [10], pp. 174 for details).

For the remaining part of this section we assume that $G$ has only one conjugacy class of Cartan subgroups. Given $f$ in $L^{1}(G)$, we can define the group Fourier transform on $\widehat{M} \times \mathcal{A}^{*}$ by

$$
\hat{f}(\xi, \lambda)=\hat{f}\left(\pi_{\xi, \lambda}\right)=\pi_{\xi, \lambda}(f)=\int_{G} f(x) \pi_{\xi, \lambda}(x) d x
$$

for $(\xi, \lambda) \in \widehat{M} \times \mathcal{A}^{*}$ (-the integral being interpreted suitably). If $f \in L^{1}(G) \cap$ $L^{2}(G)$, we have the Plancherel theorem for such $G$ : There exists an explicitly computable measure $\mu$ on $\widehat{M} \times \mathcal{A}^{*}$ such that

$$
\int_{G}|f(x)|^{2} d x=\int_{\widehat{M} \times \mathcal{A}^{*}} \operatorname{tr}\left(\pi_{\xi, \lambda}(f) \pi_{\xi, \lambda}(f)^{*}\right) d \mu(\xi, \lambda) .
$$

For fixed $\xi \in \widehat{M}$, this measure is of at most polynomial growth on $\mathcal{A}^{*}$ (see $[\mathbf{1 0}]$, pp. 511 and $[6]$ for details). Let $\mathcal{A}_{\mathbb{C}}^{*}=\mathcal{A}^{*} \otimes \mathbb{C}$. Since $B$ is positive definite on $\mathcal{A}$, it defines an inner product on $\mathcal{A}$. Hence there is a natural inner product on $\mathcal{A}^{*}$, and the corresponding norm on $\mathcal{A}^{*}$ will be denoted by $\|\cdot\|$. This real inner product can be extended in a unique fashion as an inner product on the complex vector space $\mathcal{A}_{\mathbb{C}}^{*}$ and the corresponding norm on $\mathcal{A}_{\mathbb{C}}^{*}$ will also be denoted by $\|\cdot\|$. By abuse of notation, the norm induced by $B$ on $\mathcal{A}$ will also be denoted by $\|\cdot\|$.

If 1 is the trivial representation in $\widehat{M}$, then we denote $\pi_{1, \lambda}$ by $\pi_{\lambda}$. The set of representations $\left\{\pi_{\lambda}\right\}_{\lambda \in \mathcal{A}^{*}}$ are called the class -1 principal series representations of $G$, and they are realized on the Hilbert space $L^{2}(\mathrm{~K} / \mathrm{M})$. Let $\Phi_{\lambda}$ be the "elementary spherical function" corresponding to $\lambda \in \mathcal{A}_{\mathbb{C}}^{*}$. Then for $\lambda \in \mathcal{A}^{*}$,

$$
\Phi_{\lambda}(x)=\left\langle\pi_{\lambda}(x) 1,1\right\rangle, \quad x \in G
$$


where 1 is the constant function 1 on $\mathrm{K} / \mathrm{M}$. Also one has;

$$
\begin{aligned}
\Phi_{\lambda}(x) & =\int_{K} e^{-(\imath \lambda+\rho)\left(H\left(x^{-1} k\right)\right)} d k \\
& =\int_{K} e^{(\imath \lambda-\rho)(H(x k))} d k
\end{aligned}
$$

for $\lambda \in \mathcal{A}_{\mathbb{C}}^{*}\left(\cong \mathbb{C}^{l}\right)$. Moreover for $\lambda \in \mathcal{A}^{*}\left(\cong \mathbb{R}^{l}\right)$ and any $a \in \overline{A^{+}}$, we have the following estimate:

$$
\left|\Phi_{\imath \lambda}(a)\right| \leq e^{\lambda^{+}(\log a)}
$$

where $\lambda^{+}$is the element in the fundamental Weyl chamber corresponding to $\lambda$ (see [4] for details).

Finally, we end this section with a lemma from complex analysis that is crucial for the proof of our main theorem. We shall also denote the standard Euclidean norms on $\mathbb{R}^{n}$ and $\mathbb{C}^{n}$ by $\|\cdot\|$.

Lemma 2.1. Let $n \geq 1$. Let $h$ be an entire function on $\mathbb{C}^{n}$ such that

$$
\begin{aligned}
& |h(z)| \leq C e^{a\|z\|^{2}}, \quad z \in \mathbb{C}^{n}, \\
& |h(t)| \leq C e^{-a\|t\|^{2}}, \quad t \in \mathbb{R}^{n},
\end{aligned}
$$

for some positive constants $a$ and $C$. Then $h(z)=$ Const. $e^{-a\left(z_{1}^{2}+\cdots+z_{n}^{2}\right)}, z=$ $\left(z_{1}, \cdots, z_{n}\right) \in \mathbb{C}^{n}$.

Proof. To prove this, we will need the following lemma from [17] (pp. 175):

Lemma $(*)$. Let $h$ be an entire function on $\mathbb{C}$ such that $h(z)=O\left(e^{a|z|}\right)$ for $z \in \mathbb{C}$ and $h(t)=O\left(e^{-a t}\right)$ for $t \in \mathbb{R}^{+}$, where $a$ is a positive constant. Then $h(z)=$ Const. $e^{-a z}, z \in \mathbb{C}$.

We shall prove Lemma (2.1) in two steps. First, we prove the lemma for the case $n=1$, and then proceed to prove it in general.

Let $h$ be an entire function on $\mathbb{C}$ satisfying the following estimates:

$$
\begin{gathered}
|h(z)| \leq C e^{a|z|^{2}}, \quad z \in \mathbb{C}, \\
|h(t)| \leq C e^{-a t^{2}}, \quad t \in \mathbb{R},
\end{gathered}
$$

for some positive constants $a$ and $C$. If $h$ is even, then by applying (*) to $\phi(z)=h(\sqrt{z})$, the result will follow immediately. (Note that since $h$ 
is even and entire $\phi(z)=h(\sqrt{z})$ is an entire function and will satisfy the assumptions of $(*)$.)

Suppose $h$ is an odd, entire function and $h$ satisfies (2.9) and (2.10). Then the function $\phi(z)=h(z) / z$ is an even, entire function on $\mathbb{C}$ satisfying the estimates (2.9) and (2.10). Therefore, by the even case, we have, $\phi(z)=$ $h(z) / z=C^{\prime} e^{-a z^{2}}, z \in \mathbb{C}$, for some constant $C^{\prime}$. In particular, $h(t)=$ $C^{\prime} t e^{-a t^{2}}, t \in \mathbb{R}$. Then by (2.10) it will follow that:

$$
\left|C^{\prime} t e^{-a t^{2}}\right| \leq C e^{-a t^{2}}, t \in \mathbb{R}
$$

which is impossible, unless $C^{\prime}=0$. Hence $h \equiv 0$.

If $h$ is an entire function on $\mathbb{C}$ satisfying the estimates (2.9) and (2.10), then write $h(z)=(h(z)+h(-z)) / 2+(h(z)-h(-z)) / 2=h_{\text {even }}(z)+h_{\text {odd }}(z)$, as the sum of even and odd entire functions. Since $h$ satisfies (2.9) and (2.10), it is easy to see, in view of the expressions for $h_{\text {even }}$ and $h_{\text {odd }}$, that they also satisfy (2.9) and (2.10) respectively. Applying the even and odd cases to $h_{\text {even }}$ and $h_{\text {odd }}$ respectively, we conclude that $h(z)=$ Const. $e^{-a z^{2}}, z \in \mathbb{C}$. This proves the lemma in the case when $n=1$.

Now consider the case $n>1$. For fixed $\left(u_{1}, \cdots, u_{n-1}\right)$ in $\mathbb{R}^{n-1}$, let $g(z)=$ $h\left(u_{1}, \cdots, u_{n-1}, z\right), z \in \mathbb{C}$. Clearly, $g$ is an entire function on $\mathbb{C}$ in the variable $z$. Since $h$ satisfies $(2.7)$ and (2.8), for fixed $\left(u_{1}, \cdots, u_{n-1}\right) \in \mathbb{R}^{n-1}$, we have:

$$
\begin{aligned}
& |g(z)|=\left|h\left(u_{1}, \cdots, u_{n-1}, z\right)\right| \leq C e^{a\left(\left|u_{1}\right|^{2}+\cdots+\left|u_{n-1}\right|^{2}\right)} e^{a|z|^{2}}, \quad z \in \mathbb{C}, \\
& |g(t)|=\left|h\left(u_{1}, \cdots, u_{n-1}, t\right)\right| \leq C e^{-a\left(\left|u_{1}\right|^{2}+\cdots+\left|u_{n-1}\right|^{2}\right)} e^{-a t^{2}}, \quad t \in \mathbb{R} .
\end{aligned}
$$

Applying the one dimensional case to $g$ we can conclude that

$$
g(z)=C_{n}\left(u_{1}, \cdots, u_{n-1}\right) e^{-a z^{2}}, z \in \mathbb{C},\left(u_{1}, \cdots, u_{n-1}\right) \in \mathbb{R}^{n-1},
$$

where $C_{n}$ depends only on $u_{1}, \cdots, u_{n-1}$. Setting $z=0$, we have

$$
C_{n}\left(z_{1}, \cdots, z_{n-1}\right)=g(0)=h\left(z_{1}, \cdots, z_{n-1}, 0\right)
$$

for $\left(z_{1}, \cdots, z_{n-1}\right) \in \mathbb{R}^{n-1}$. Thus

$$
h\left(z_{1}, \cdots, z_{n-1}, z_{n}\right)=h\left(z_{1}, \cdots, z_{n-1}, 0\right) e^{-a z_{n}^{2}}
$$

for all $\left(z_{1}, \cdots, z_{n-1}, z_{n}\right) \in \mathbb{R}^{n}$. However, both sides are entire functions on $\mathbb{C}^{n}$ and hence (2.11) must actually hold for all $\left(z_{1}, \cdots, z_{n-1}, z_{n}\right) \in \mathbb{C}^{n}$. Here we are using the fact that two entire functions on $\mathbb{C}^{n}$ which agree on $\mathbb{R}^{n}$ have to actually agree on $\mathbb{C}^{n}$. Now from $(2.7)$ and (2.8) it follows that

$$
\left.h\left(z_{1}, \cdots, z_{n-1}, 0\right)=O\left(e^{a\left(\left|z_{1}\right|^{2}+\cdots+\left|z_{n-1}\right|^{2}\right.}\right)\right),\left(z_{1}, \cdots, z_{n-1}\right) \in \mathbb{C}^{n-1},
$$


and

$$
h\left(t_{1}, \cdots, t_{n-1}, 0\right)=O\left(e^{-a\left(\left|t_{1}\right|^{2}+\cdots+\left|t_{n-1}\right|^{2}\right)}\right),\left(t_{1}, \cdots, t_{n-1}\right) \in \mathbb{R}^{n-1},
$$

and applying exactly the same argument as before we will have

$$
h\left(z_{1}, \cdots, z_{n-1}, 0\right)=h\left(z_{1}, \cdots, z_{n-2}, 0,0\right) e^{-a z_{n-1}^{2}},
$$

and so

$$
h\left(z_{1}, \cdots, z_{n}\right)=h\left(z_{1}, \cdots, z_{n-2}, 0,0\right) e^{-a\left(z_{n-1}^{2}+z_{n}^{2}\right)} .
$$

Repeating the above, we finally have

$$
h\left(z_{1}, \cdots, z_{n}\right)=h(0,0, \cdots, 0) e^{-a\left(z_{1}^{2}+\cdots+z_{n}^{2}\right)},\left(z_{1}, \cdots, z_{n}\right) \in \mathbb{C}^{n},
$$

and the proof of the lemma is complete.

In the next section we will state and prove an analogue of Hardy's theorem for a class of semi-simple Lie groups.

\section{Semi-simple Lie groups with one conjugacy class of Cartan subgroups.}

We retain the notation introduced in Section 2. However we assume that $G$ has only one conjugacy class of Cartan subgroups. Thus, throughout this section, $G$ will denote a connected non-compact semi-simple Lie group with finite centre and having only one conjugacy class of Cartan subgroups. For such groups, as described in Section 2, the Plancherel measure is entirely supported on the various principal series representations associated with the minimal parabolic. We now state and prove an analogue of Hardy's theorem for such groups.

Theorem 3.1. $\quad$ Suppose $f$ is a measurable function on $G$ satisfying the following estimates:

$$
\begin{gathered}
|f(x)| \leq C e^{-\alpha\|x\|_{G}^{2}}, \quad x \in G \\
\|\hat{f}(\xi, \lambda)\|_{H S}=\left\|\pi_{\xi, \lambda}(f)\right\|_{H S} \leq C_{\xi} e^{-\beta\|\lambda\|^{2}}, \quad(\xi, \lambda) \in \widehat{M} \times \mathcal{A}^{*}
\end{gathered}
$$

where $C, C_{\xi}, \alpha$ and $\beta$ are positive constants and $C_{\xi}$ depends on $\xi$. If $\alpha \beta>\frac{1}{4}$, then $f=0$ a.e.

(Note: (i) The very rapid decay of $f$ implies $f \in L^{1}(G)$. Hence $\pi_{\xi, \lambda}(f)$ is defined for all $\xi \in \widehat{M}, \lambda \in \mathcal{A}^{*}$. Here $\|T\|_{H S}$ denotes the Hilbert-Schmidt 
norm of $T$.

(ii) If $x=k_{1} a k_{2}, a=\exp H$, then $\|x\|_{G}=\|H\|$, where $\|\cdot\|$ is the norm on $\mathcal{A}$ described in Section 2.)

Proof. For $\xi \in \widehat{M}$ let $\left\{e_{j}^{\xi}: j \in \mathbb{N}\right\}$ be a basis of $\mathbb{H}_{\xi}^{o}$ consisting of $K$-finite vectors. (As observed earlier the action of $K$ on $\mathbb{H}_{\xi}^{o}$ is just left regular action.) Let $\langle\cdot, \cdot\rangle_{\xi}$ denote the inner product on $\mathbb{H}_{\xi}^{o}$. We shall show that if $\alpha \beta>\frac{1}{4}$, $\left\langle\pi_{\xi, \lambda}(f) e_{m}^{\xi}, e_{n}^{\xi}\right\rangle_{\xi}=0$, for all $\lambda \in \mathcal{A}^{*}, m, n \in \mathbb{N}$. Fix $m_{o}, n_{o} \in \mathbb{N}$. We have by $(2.2)$ :

$$
\left\langle\pi_{\xi, \lambda}(f) e_{m_{o}}^{\xi}, e_{n_{o}}^{\xi}\right\rangle_{\xi}=\int_{G} f(x)\left\langle\pi_{\xi, \lambda}(x) e_{m_{o}}^{\xi}, e_{n_{o}}^{\xi}\right\rangle_{\xi} d x
$$

Let $\Phi_{\xi, \lambda}^{m_{o}, n_{o}}(x)=\left\langle\pi_{\xi, \lambda}(x) e_{m_{o}}^{\xi}, e_{n_{o}}^{\xi}\right\rangle_{\xi}$ for $x \in G$. Then it can be shown from the definition of $\pi_{\xi, \lambda}(x)$ acting on $\mathbb{H}_{\xi}^{o}$ that:

$$
\Phi_{\xi, \lambda}^{m_{o}, n_{o}}(x)=\int_{K} e^{-(\imath \lambda+\rho)\left(H\left(x^{-1} k\right)\right)}\left\langle e_{m_{o}}^{\xi}\left(\kappa\left(x^{-1} k\right)\right), e_{n_{o}}^{\xi}(k)\right\rangle d k
$$

where $\langle\cdot, \cdot\rangle$ inside the integral is the inner product on $\mathbb{H}_{\xi}$. Thus

$$
\left\langle\pi_{\xi, \lambda}(f) e_{m_{o}}^{\xi}, e_{n_{o}}^{\xi}\right\rangle_{\xi}=\int_{G} f(x) \Phi_{\xi, \lambda}^{m_{o}, n_{o}}(x) d x
$$

The basis vectors $e_{m_{o}}^{\xi}, e_{n_{o}}^{\xi}$ being $K$-finite, actually belong to $C^{\infty}\left(K, \mathbb{H}_{\xi}\right)$ and hence are bounded as functions into $\mathbb{H}_{\xi}$. Therefore it follows easily that for each $x \in G$, the integral defining $\Phi_{\xi, \lambda}^{m_{o}, n_{o}}$ makes sense even for $\lambda \in \mathcal{A}_{\mathbb{C}}^{*}$ and in fact, for each fixed $x$, the function $\lambda \mapsto \Phi_{\xi, \lambda}^{m_{o}, n_{o}}(x)$ extends as an entire function of $\lambda \in \mathcal{A}_{\mathbb{C}}^{*}\left(\cong \mathbb{C}^{l}\right)$. Writing $\lambda=\lambda_{R}+\imath \lambda_{I}$, one has the following easy estimate from the above integral:

$$
\left|\Phi_{\xi, \lambda}^{m_{o}, n_{o}}(x)\right| \leq \text { Const. } \int_{K} e^{\left(\lambda_{I}-\rho\right)\left(H\left(x^{-1} k\right)\right)} d k
$$

where the constant depends only on $m_{o}, n_{o}$ and $\xi$. The integral on the right is just the elementary spherical function $\Phi_{\imath \lambda_{I}}$ and hence we have the following easy estimate

$$
\left|\Phi_{\xi, \lambda}^{m_{o}, n_{o}}(x)\right| \leq \text { Const. } \Phi_{\imath \lambda_{I}}(x)
$$

Using the $K$-biinvariance of $\Phi_{\imath \lambda_{I}}$, one therefore finally has, if $x$ is written as $x=k_{1} a k_{2}, k_{1}, k_{2} \in K, a \in \overline{A^{+}}$,

$$
\left|\Phi_{\xi, \lambda}^{m_{o}, n_{o}}(x)\right| \leq \text { Const. } e^{\lambda_{I}^{+}(\log a)}
$$


where $\lambda_{I}^{+}$is the element in the fundamental Weyl chamber corresponding to $\lambda_{I}$ (see [4] for details). Now define

$$
G(\lambda)=\int_{G} \Phi_{\xi, \lambda}^{m_{o}, n_{o}}(x) f(x) d x, \quad \lambda \in \mathcal{A}^{*} .
$$

Then $G(\lambda)=\left\langle\pi_{\xi, \lambda}(f) e_{m_{o}}^{\xi}, e_{n_{o}}^{\xi}\right\rangle_{\xi}$ for $\lambda \in \mathcal{A}^{*}$. Also observe that as $f$ decays very rapidly (3.1), the analyticity of $\lambda \mapsto \Phi_{\xi, \lambda}^{m_{o}, n_{o}}(x)$ on $\mathcal{A}_{\mathbb{C}}^{*}\left(\cong \mathbb{C}^{l}\right)$ for each fixed $x \in G$, the estimate (3.8) together with (3.5) will imply that the integral defining the function $G(\lambda)$ makes sense for $\lambda \in \mathcal{A}_{\mathbb{C}}^{*}$ and in fact defines an entire function. Moreover, for $\lambda=\lambda_{R}+\imath \lambda_{I} \in \mathcal{A}_{\mathbb{C}}^{*}$,

$$
|G(\lambda)| \leq \int_{G}|f(x)|\left|\Phi_{\xi, \lambda}^{m_{o}, n_{o}}(x)\right| d x .
$$

Now using polar coordinates, (3.8) and the fact that if $x=k_{1} a k_{2},\|x\|_{G}=$ $\|a\|_{G}$, the integral on the right hand side is majorized by

$$
\text { Const. } \int_{\overline{A^{+}}} e^{-\alpha\|a\|_{G}^{2}} e^{\lambda_{I}^{+}(\log a)}|J(a)| d a,
$$

where $d a$ denotes the Haar measure on $A$. If $H \in \mathcal{A}$ is the unique element such that $\exp H=a$ and $d H$ denotes the Lebesgue measure on $\mathcal{A}$, then it can be easily seen that there exists a constant $C$ such that $|J(a)| \leq$ Const. $e^{C\|H\|}$ and the integral on the right hand side is majorized by Const. $\int_{\mathcal{A}} e^{-\alpha\|H\|^{2}} e^{\lambda_{I}^{+}(H)} e^{C\|H\|} d H$, where now $\|\cdot\|$ is the norm on $\mathcal{A}$ induced by the Cartan-Killing form.

Now let $H_{\lambda_{I}}$ be the unique element of $\mathcal{A}$ such that $\lambda_{I}^{+}(H)=\left\langle H, H_{\lambda_{I}}\right\rangle$ for all $H$, where $\langle\cdot, \cdot\rangle$ is the inner product on $\mathcal{A}$ induced by the Cartan-Killing form. Then there exists $0<\alpha^{\prime}<\alpha$ such that we continue to have $\alpha^{\prime} \beta>\frac{1}{4}$ and $e^{-\alpha\|H\|^{2}+C\|H\|} \leq$ Const. $e^{-\alpha^{\prime}\|H\|^{2}}$. So:

$$
\begin{aligned}
\int_{\mathcal{A}} e^{-\alpha\|H\|^{2}} e^{\lambda_{I}^{+}(H)} e^{C\|H\|} d H \\
\quad=\int_{\mathcal{A}} e^{-\alpha\|H\|^{2}+\left\langle H, H_{\lambda_{I}}\right\rangle+C\|H\|} d H \\
\quad \leq \text { Const. } \int_{\mathcal{A}} e^{-\alpha^{\prime}\|H\|^{2}+\left\langle H, H_{\lambda_{I}}\right\rangle} d H \\
\quad=\text { Const. } e^{\frac{1}{4 \alpha^{\prime}}\left\|H_{\lambda_{I}}\right\|^{2}} \int_{\mathcal{A}} e^{-\alpha^{\prime}\left\langle H-\frac{1}{2 \alpha^{\prime}} H_{\lambda_{I}}, H-\frac{1}{2 \alpha^{\prime}} H_{\lambda_{I}}\right\rangle} d H \\
\quad=\text { Const. } e^{\frac{1}{4 \alpha^{\prime}}\left\|H_{\lambda_{I}}\right\|^{2}} \int_{\mathcal{A}} e^{-\alpha^{\prime}\|H\|^{2}} d H
\end{aligned}
$$

(by translation invariance of Lebesgue measure). But by the choice of inner product on $\mathcal{A}^{*},\left\|H_{\lambda_{I}}\right\|=\left\|\lambda_{I}^{+}\right\|$. Further the action of the Weyl group preserves the norm on $\mathcal{A}^{*}$ and hence $\left\|\lambda_{I}^{+}\right\|=\left\|\lambda_{I}\right\| \leq\|\lambda\|$. So finally we get the 
estimate

$$
|G(\lambda)| \leq C e^{\frac{1}{4 \alpha^{\prime}}\|\lambda\|^{2}}, \quad \lambda \in \mathcal{A}_{\mathbb{C}}^{*} \cong \mathbb{C}^{l}
$$

for some constant $C$. But for $\lambda \in \mathcal{A}^{*}$ by (3.2),

$$
|G(\lambda)| \leq C_{\xi} e^{-\beta\|\lambda\|^{2}}
$$

Since $\alpha^{\prime} \beta>\frac{1}{4},-\beta<-\frac{1}{4 \alpha^{\prime}}$ and so we have $|G(\lambda)| \leq C e^{\frac{1}{4 \alpha^{\prime}}\|\lambda\|^{2}}$ for $\lambda \in \mathcal{A}_{\mathbb{C}}^{*}$ and $|G(\lambda)| \leq C_{\xi} e^{-\frac{1}{4 \alpha^{\prime}}\|\lambda\|^{2}}$ for $\lambda \in \mathcal{A}^{*}$. So by Lemma (2.1), we have $G(\lambda)=$ Const. $e^{-\frac{1}{4 \alpha^{*}}\|\lambda\|^{2}}, \lambda \in \mathcal{A}^{*}$. Therefore we have for $\lambda \in \mathcal{A}^{*}$,

$$
\mid \text { Const. } e^{-\frac{1}{4 \alpha^{\prime}}\|\lambda\|^{2}}|=| G(\lambda) \mid \leq C_{\xi} e^{-\beta\|\lambda\|^{2}} .
$$

But $\beta-\frac{1}{4 \alpha^{\prime}}>0$ and hence we would have

$$
\mid \text { Const. } e^{\left(\beta-\frac{1}{4 \alpha^{\prime}}\right)\|\lambda\|^{2}} \mid \leq C_{\xi}, \quad \lambda \in \mathcal{A}^{*}
$$

and this is impossible unless the constant on the left hand side is zero i.e. $G(\lambda) \equiv 0$ i.e. for arbitrary $\xi \in \widehat{M}, m_{o}, n_{o} \in \mathbb{N},\left\langle\pi_{\xi, \lambda}(f) e_{m_{o}}^{\xi}, e_{n_{o}}^{\xi}\right\rangle_{\xi} \equiv 0$ as a function of $\lambda$. Hence it follows that $\pi_{\xi, \lambda}(f) \equiv 0$ on $\widehat{M} \times \mathcal{A}^{*}$ and since the Plancherel measure is supported on $\widehat{M} \times \mathcal{A}^{*}$, it follows that $f=0$ a.e.

\section{Arbitrary semi-simple Lie groups.}

We continue to retain the notation introduced in Section 2. In this section, $G$ will denote an arbitrary noncompact semi-simple Lie group with finite centre i.e. we drop the assumption that $G$ has only one conjugacy class of Cartan subgroups. Instead, we impose some restrictions on the kind of functions being considered; we will consider only right $K$-invariant functions. For the harmonic analysis of such functions, only the class- 1 principal series representations are relevant. Let $\left\{\pi_{\lambda}\right\}_{\lambda \in \mathcal{A}^{*}}$ denote the class-1 principal series representations of $G$ (i.e. $\pi_{\lambda}=\pi_{1, \lambda}$ where 1 is the trivial representation of $M)$. These can all be realized on $L^{2}(\mathrm{~K} / \mathrm{M})$. Let $v_{o}$ be the constant function 1 on $\mathrm{K} / \mathrm{M}$ i.e. $v_{o}$ is the essentially unique $K$-fixed vector in $L^{2}(\mathrm{~K} / \mathrm{M})$ for the representation $\pi_{\lambda}$. Then one knows that if $v$ is any other $K$-finite vector in $L^{2}(\mathrm{~K} / \mathrm{M})$ which is not a multiple of $v_{o}$, then $\pi_{\lambda}(f) v=0$. Thus $\pi_{\lambda}(f)$ is completely determined by $\pi_{\lambda}(f) v_{o}$ and moreover $\left\|\pi_{\lambda}(f)\right\|_{H S}=\left\|\pi_{\lambda}(f) v_{o}\right\|$, where $\|\cdot\|$ denotes the usual norm in $L^{2}(\mathrm{~K} / \mathrm{M})$. Thus the group theoretic Fourier transform can be thought of as a function on $\mathcal{A}^{*}$ alone, taking values in the Hilbert space $L^{2}(\mathrm{~K} / \mathrm{M})$. Keeping these considerations in mind, an examination of the proof of Theorem (3.1) immediately yields the following result: 
Theorem 4.1. Suppose $f$ is a measurable right $K$-invariant function on $G$ (i.e. $f(x k)=f(x), x \in G, k \in K)$, satisfying the following estimates for some positive constants $C, \alpha$ and $\beta$ :

$$
\begin{aligned}
&|f(x)| \leq C e^{-\alpha\|x\|_{G}^{2}}, x \in G, \\
&\left\|\pi_{\lambda}(f) v_{o}\right\| \leq C e^{-\beta\|\lambda\|^{2}}, \quad \lambda \in \mathcal{A}^{*} .
\end{aligned}
$$

If $\alpha \beta>\frac{1}{4}$, then $f=0$ a.e.

One can view the above as a theorem about functions on $\mathrm{G} / \mathrm{K}$, which is a symmetric space of the noncompact type; the group theoretic Fourier transform can be reinterpreted as the Fourier transform on the symmetric space, as introduced by Helgason (see [8]). A brief discussion from this point of view can be found in [12].

\section{Further remarks.}

5.1. $S L(2, \mathbb{R})$. Thus in Section 3 , we have established an analogue of Hardy's theorem for a class of semi-simple Lie groups which include all complex groups and real rank-1 groups without Discrete Series representations. However we would like to conjecture that a result of a similar nature is valid for all noncompact semi-simple Lie groups. For instance, in the case when $G=S L(2, \mathbb{R})$, we shall prove the exact analogue of Theorem (3.1). For this we need to recall some facts from the representation theory of $S L(2, \mathbb{R})$. The reader can find the details of the material covered in this section in $[\mathbf{3}],[\mathbf{1 1}]$ and $[14]$.

In this section, we shall continue to use the notation introduced in Section 2 except that the norm defined on $\mathcal{A}^{*}$ in Section 2 is denoted by $\|\cdot\|_{\mathcal{A}^{*}}$.

For the time being, let $G=S L(2, \mathbb{R})$. Then

$$
K=S O(2)\left(\simeq S^{1}\right), \quad \mathcal{A}=\left\{\left(\begin{array}{c}
t 0 \\
0-t
\end{array}\right): t \in \mathbb{R}\right\} \quad \text { and } M=\left\{ \pm\left(\begin{array}{l}
10 \\
01
\end{array}\right)\right\} .
$$

Therefore

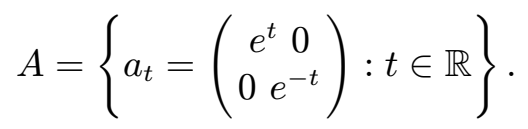

The polar decomposition of an element $g \in G$ can be written as

$$
g=k_{\theta_{1}} a_{t} k_{\theta_{2}} \text { where } k_{\theta}=\left(\begin{array}{cc}
\cos \theta & \sin \theta \\
-\sin \theta & \cos \theta
\end{array}\right) .
$$

In this case, there are only two irreducible representations of $M$. Corresponding to the two irreducible representations of $M$, one gets two sets of 
principal series representations denoted by $\pi_{1, \lambda}, \pi_{-1, \lambda}$ of $G$, defined exactly as in Section 2. Each set of principal series representations is parametrized by $\mathcal{A}^{*} \simeq \mathbb{R}$. The series $\pi_{1, \lambda}$ is irreducible for all $\lambda \in \mathbb{R}$ and $\pi_{-1, \lambda}$ is irreducible for all $\lambda \in \mathbb{R} \backslash\{0\}$. There is another set of irreducible, unitary representations of $G$ called the Discrete series. For each $n \in \mathbb{Z},|n| \geq 2$, denote by $D_{n}$ the corresponding discrete series representation of $G$. For details about the $K$-module structure and the spaces on which these representations are realized, see [11], [14] etc. (Apart from these, there is another collection of irreducible, unitary representations of $G$, called the complementary series, which do not play a role in the Plancherel measure.) The Plancherel measure $\mu$ is supported on the set of principal and discrete series representations (see [11] for details).

For $n \in \mathbb{Z}$, define $\chi_{n}$ on $S O(2)\left(\simeq S^{1}\right)$ by $\chi_{n}\left(k_{\theta}\right)=e^{\imath n \theta}$. Let $\pi$ be an irreducible unitary representation of $G$ on the Hilbert space $\mathbb{H}_{\pi}$. If $0 \neq v \in$ $\mathbb{H}_{\pi}$ is such that $\pi\left(k_{\theta}\right) v=\chi_{l}\left(k_{\theta}\right) v, l \in \mathbb{Z}, k_{\theta} \in K$, then we say " $v$ transforms according to $\chi_{l}$ ". If such a non-zero $v$ exists then it is unique upto scalar multiplication (see [3]) and we say that " $\chi_{l}$ occurs in $\pi$ ". If $m$ and $n$ are fixed integers such that $\chi_{m}$ and $\chi_{n}$ occur in $\pi$, let $v_{m}, v_{n}$ be the essentially unique unit vectors transforming according to $\chi_{m}$ and $\chi_{n}$ respectively. Denote by $\Phi_{\pi}^{m, n}$ the function defined by $\Phi_{\pi}^{m, n}(g)=\left\langle\pi(g) v_{n}, v_{m}\right\rangle$ - this is the "elementary spherical function of type $(\mathrm{m}, \mathrm{n})$ corresponding to $\pi$ ". Here $\langle\cdot, \cdot\rangle$ denotes the inner product on $\mathbb{H}_{\pi}$. If $D_{l}$ is a Discrete series representation of $G$ and $\chi_{m}$, $\chi_{n}$ occur in $D_{l}$, then explicit formulae are available for $\Phi_{D_{l}}^{m, n}$ (see [3] and [14]). Denote $\Phi_{D_{l}}^{m, n}$ by $\Phi_{l}^{m, n}$. It turns out that for $a_{t} \in A, \Phi_{l}^{m, n}\left(a_{t}\right)$ is a rational function of $e^{-t}$ such that as $t \rightarrow \infty, \Phi_{l}^{m, n}\left(a_{t}\right) \rightarrow 0$. (Note that $e^{-t} \rightarrow 0$ as $t \rightarrow \infty$ !)

Now we are in a position to state and prove the exact analogue of Theorem (3.1) for $G=S L(2, \mathbb{R})$ :

Theorem 5.1. Suppose $f$ is a measurable function on $G$ satisfying the following estimates:

$$
\begin{gathered}
|f(x)| \leq C e^{-\alpha\|x\|_{G}^{2}}, \quad x \in G, \\
\left\|\pi_{\xi, \lambda}(f)\right\|_{H S} \leq C e^{-\beta\|\lambda\|_{\mathcal{A}^{*}}^{2}}, \quad \xi \in \widehat{M}, \lambda \in \mathcal{A}^{*},
\end{gathered}
$$

where $C, \alpha, \beta$ are positive constants. If $\alpha \beta>\frac{1}{4}$, then $f=0$ a.e.

Proof. Any $f \in L^{1}(G)$ can be written (in the sense of distributions) as $f \approx \sum_{m, n \in \mathbb{Z}} \chi_{m} * f * \chi_{n}=\sum_{m, n \in \mathbb{Z}} f_{m n}$. Note that each $f_{m n}$ has the property that

$$
f_{m n}\left(k_{\theta_{1}} g k_{\theta_{2}}\right)=\chi_{m}\left(k_{\theta_{1}}\right) f(g) \chi_{n}\left(k_{\theta_{2}}\right)
$$


$k_{\theta_{1}}, k_{\theta_{2}} \in K$. One can easily show that if $f$ satisfies estimates (5.1) and (5.2), then so does each $f_{m n}$. Clearly, if each $f_{m n}=0$, then so is $f$. Hence, without loss of generality, assume that $f$ satisfies (5.3) for some fixed $m, n \in \mathbb{Z}$. Then exactly as in the proof of Theorem (3.1), it will follow that $\pi_{1, \lambda}(f) \equiv 0$ and $\pi_{-1, \lambda}(f) \equiv 0$ as functions of $\lambda$. Thus by the inversion formula (see [11] and [14]) for functions that satisfy (5.3), it follows that $f$ is a linear combination of elementary spherical functions of type $(m, n)$ of finitely many Discrete series representations. (Note that for fixed $m, n \in \mathbb{Z}$, there are only finitely many Discrete series representations in which $\chi_{m}$ and $\chi_{n}$ occur.) Since each $\Phi_{l}^{m, n}$ evaluated at $a_{t}=\left(\begin{array}{cc}e^{t} & 0 \\ 0 & e^{-t}\end{array}\right) \in G, t>0$, is a rational function of $e^{-t}$, it follows that $f\left(a_{t}\right)$ is a rational function of $e^{-t}$. Also as noted, when $t \rightarrow \infty$ i.e. $e^{-t} \rightarrow 0$, each $\Phi_{l}^{m, n} \rightarrow 0$. So $f\left(a_{t}\right) \rightarrow 0$ as $e^{-t} \rightarrow 0$.

Suppose $f\left(a_{t}\right) \not \equiv 0$ as a function of $t$. We will arrive at a contradiction. Since $f\left(a_{t}\right)$ is a rational function of $e^{-t}, f\left(a_{t}\right)=\left(e^{-t}\right)^{l} g\left(e^{-t}\right)$ for some positive integer $l$, where $g\left(e^{-t}\right)$ is also a rational function of $e^{-t}$ and coverges to a finite non zero limit $\gamma$ as $t \rightarrow \infty$ (i.e. $e^{-t} \rightarrow 0$ ). On the other hand

$$
\left|f\left(a_{t}\right)\right| \leq C e^{-\alpha^{\prime} t^{2}}
$$

where $\alpha^{\prime}$ is a positive constant depending on $\alpha$ and the way the norm is defined on $\mathcal{A}$. Hence we would have

$$
\left|\left(e^{-t}\right)^{l} g\left(e^{-t}\right)\right| \leq C e^{-\alpha^{\prime} t^{2}}
$$

as $t \rightarrow \infty$. But since $g\left(e^{-t}\right) \rightarrow \gamma$, and $\gamma$ is non zero, this clearly leads to a contradiction. This completes the proof of the theorem.

5.2. The sharpness of the constant $\frac{1}{4}$. For the group $G=S L(2, \mathbb{C})$, using the normalizations in this paper, we will show that $\frac{1}{4}$ is the best possible constant. First we recall a couple of facts. If $f$ is an $L^{1}$-function invariant under the right action of $K$, then $\pi_{\xi, \lambda}(f)=0$ unless $\xi$ is the trivial representation of $M$. Thus, for such functions, it is enough to consider $\left\{\pi_{1, \lambda}\right\}_{\lambda \in \mathcal{A}^{*}}$. As before, denote $\pi_{1, \lambda}$ by $\pi_{\lambda}$. Now let $v_{o}$ be the essentially unique $K$-fixed vector in $L^{2}(\mathrm{~K} / \mathrm{M})$ for the representation $\pi_{\lambda}$. ( $v_{o}$ is the constant function 1 on $\mathrm{K} / \mathrm{M}$.) Then, as observed in Section 4 , for such $f,\left\|\pi_{\lambda}(f)\right\|_{H S}=\left\|\pi_{\lambda}(f) v_{o}\right\|$, where $\|\cdot\|$ denotes the usual norm in $L^{2}(\mathrm{~K} / \mathrm{M})$. Further, if $f$ is also left $K$ invariant i.e. $f$ is $K$-biinvariant, then $\pi_{\lambda}(f) v_{o}=\left\langle\pi_{\lambda}(f) v_{o}, v_{o}\right\rangle v_{o}$ and hence $\left\|\pi_{\lambda}(f)\right\|_{H S}=\left|\left\langle\pi_{\lambda}(f) v_{o}, v_{o}\right\rangle\right|$. Now $\left\langle\pi_{\lambda}(f) v_{o}, v_{o}\right\rangle=\int_{G}\left\langle\pi_{\lambda}(x) v_{o}, v_{o}\right\rangle f(x) d x$, where $d x$ is the Haar measure on $G$. So, as before, if we denote the function $x \mapsto\left\langle\pi_{\lambda}(x) v_{o}, v_{o}\right\rangle$ by $\Phi_{\lambda}(x)$, we need to consider only the integral $\int_{G} f(x) \Phi_{\lambda}(x) d x$. The collection $\left\{\Phi_{\lambda}\right\}_{\lambda \in \mathcal{A}^{*}}$ form a subset of the set of 'elementary spherical functions' and we are actually looking at the 'spherical Fourier transform' of $f$. So let $g(\lambda)=\int_{G} f(x) \Phi_{\lambda}(x) d x$. Since $f$ is $K$ biinvariant, $f$ is completely determined by its restriction to $A$. Thus to prove 
our assertion that $\frac{1}{4}$ is the best possible constant, it is enough to produce a function $f$ which is

(a) $K$-biinvariant

(b) for every $\epsilon>0,|f(a)| \leq C_{\epsilon} e^{-\left(\frac{1}{16}-\epsilon\right)\|a\|_{G}^{2}}, a \in A$, and

(c) $|g(\lambda)|=e^{-4\|\lambda\|_{\mathcal{A}^{*}}^{2}}$, where $\|\cdot\|_{\mathcal{A}^{*}}$ is the norm on $\mathcal{A}^{*}$ induced by the Killing form.

Each $\lambda \in \mathbb{R}$ can be identified with an element in $\mathcal{A}^{*}$ via the identification

$$
\mathcal{A} \ni\left(\begin{array}{cc}
x & 0 \\
0 & -x
\end{array}\right) \mapsto \lambda x .
$$

With this identification the elementary spherical functions are given by

$$
\Phi_{\lambda}\left(\left(\begin{array}{cc}
e^{t} & 0 \\
0 & e^{-t}
\end{array}\right)\right)=\frac{2 \sin \lambda t}{\lambda \sinh 2 t}
$$

(see [16], Vol. 2, pp. 313-314). Also $\|\lambda\|_{\mathcal{A}^{*}}=\frac{|\lambda|}{4}$ and $\left\|\left(\begin{array}{cc}e^{t} & 0 \\ 0 & e^{-t}\end{array}\right)\right\|_{G}=4|t|$.

Let $g(\lambda)=e^{-\frac{\lambda^{2}}{4}}, \lambda \in \mathbb{R} \simeq \mathcal{A}^{*}$. In view of the "rapid decay" of $g$, by appealing to the Trombi-Varadarajan theorem $([\mathbf{1 8}])$, there exists a unique $K$-biinvariant $f$ such that the spherical Fourier transform of $f$ is precisely $g$.

Since in this case (i.e. $G=S L(2, \mathbb{C})$ ), the Plancherel formula and the inversion formula can be explicitly written down (see [16], Vol. 2, pp. 313314), we have

$$
\begin{aligned}
f\left(\left(\begin{array}{cc}
e^{t} & 0 \\
0 & e^{-t}
\end{array}\right)\right) & =\text { Const. } \int_{\mathbb{R}} g(\lambda) \frac{2 \sin \lambda t}{\lambda \sinh 2 t}|\lambda|^{2} d \lambda \\
& =\frac{\text { Const. }}{\sinh 2 t} \int_{\mathbb{R}} \lambda e^{-\frac{\lambda^{2}}{4}} \sin \lambda t d \lambda
\end{aligned}
$$

which is equal to Const. $\frac{t e^{-t^{2}}}{\sinh 2 t}$, using routine Euclidean Fourier transform calculations. Clearly,

$$
\left|f\left(\left(\begin{array}{cc}
e^{t} & 0 \\
0 & e^{-t}
\end{array}\right)\right)\right|=\text { Const. }\left|\frac{t}{\sinh 2 t} e^{-\frac{\left\|a_{t}\right\|_{G}^{2}}{16}}\right| \leq C_{\epsilon} e^{-\left(\frac{1}{16}-\epsilon\right)\left\|a_{t}\right\|_{G}^{2}},
$$

for each $\epsilon>0$ and $|g(\lambda)|=e^{-4\|\lambda\|^{2}} \mathcal{A}^{*}$ where $a_{t}=\left(\begin{array}{cc}e^{t} & 0 \\ 0 & e^{-t}\end{array}\right)$. Thus the fact that $\frac{1}{4}$ is the best possible constant has been established.

Acknowledgement. We thank the referee for several comments which helped in improving the exposition. 


\section{References}

[1] M. Cowling and J. Price, Generalizations of Heisenberg's inequality, Harmonic analysis, Proceedings, Cortona, Italy (1982), Mauceri G, Ricci F and Weiss G, Eds., Lecture notes No. 992, Springer-Verlag, 1983.

[2] H. Dym and H.P. McKean, Fourier series and integrals, Academic Press, New York, 1972.

[3] J.M.G. Fell, The representation theory of the real $2 \times 2$ unimodular group, Unpublished lecture notes.

[4] R. Gangolli and V.S. Varadarajan, Harmonic analysis of spherical functions on real reductive groups, Springer-Verlag, 1988.

[5] G.H. Hardy, A theorem concerning Fourier transforms, J. London Math. Soc., 8 (1933), 227-231.

[6] Harish-Chandra, The Plancherel formula for complex semi-simple Lie groups, Trans. Amer. Math. Soc., 76 (1954), 485-528.

[7] S. Helgason, Differential geometry, Lie groups, and symmetric spaces, Academic Press, 1978.

[8] _ Groups and geometric analysis, Academic Press, 1984.

[9] L. Hörmander, A uniqueness theorem of Beurling for Fourier transform pairs, Arkiv för Matematik, 29(2) (1991), 237-240.

[10] A.W. Knapp, Representation theory of semi-simple Lie groups, an overview based on examples, Princeton University Press, Princeton, 1986.

[11] S. Lang, $S L_{2}(\mathbb{R})$, Springer-Verlag, 1985.

[12] V. Pati, A. Sitaram, M. Sundari and S. Thangavelu, An uncertainty principle for eigenfunction expansions, J. Fourier Analysis and Applications, 2(5) (1996), 427433.

[13] A. Sitaram, M. Sundari and S. Thangavelu, Uncertainty principles on certain Lie groups, Proc. Indian Acad. Sci. (Math. Sci.), 105(2) (1995), 135-151.

[14] M. Sugiura, Unitary representations and harmonic analysis, An introduction, Kodansha scientific books, Tokyo, 1975.

[15] M. Sundari, Hardy's theorem for the n-dimensional Euclidean motion group, to appear in Proc. Amer. Math. Soc.

[16] A. Terras, Harmonic analysis on symmetric spaces and applications, Vols. 1 and 2, Springer-Verlag, 1988.

[17] E.C. Titchmarsh, Introduction to the theory of Fourier Integrals, Chelsea Publishing Company, New York, N.Y., 1986.

[18] P.C. Trombi and V.S. Varadarajan, Spherical transforms on semi-simple Lie groups, Ann. Math., 94 (1971), 246-303.

Received May 3, 1995 and revised November 29, 1995.

Indian Statistical Institute

8Th Mile, Mysore RoAd

BANGALORE - 560 059, INDIA

E-mail address: sitaram@isibang.ernet.in 